1 Original Research

\title{
Identification of multiple TAR DNA Binding Protein retropseudogene lineages during the evolution of primates
}

\author{
Juan C. Opazo ${ }^{1,2,3 *}$, Kattina Zavala2 , Luis Vargas-Chacoff ${ }^{1,4,5}$, Francisco J. \\ Morera $^{1,6}$, Gonzalo A. Mardones ${ }^{1,7,8^{*}}$
}

${ }^{1}$ Integrative Biology Group, Universidad Austral de Chile, Valdivia, Chile.

${ }^{2}$ Instituto de Ciencias Ambientales y Evolutivas, Facultad de Ciencias, Universidad Austral de Chile, Valdivia, Chile.

3 Millennium Nucleus of Ion Channel-Associated Diseases (MiNICAD), Valdivia, Chile.

${ }^{4}$ Instituto de Ciencias Marinas y Limnológicas, Universidad Austral de Chile, Valdivia, Chile.

${ }^{5}$ Centro Fondap de Investigación de Altas Latitudes (IDEAL), Universidad Austral de Chile, Valdivia, Chile.

${ }^{6}$ Applied Biochemistry Laboratory, Instituto de Farmacología y Morfofisiología, Facultad de Ciencias Veterinarias, Universidad Austral de Chile, Valdivia, Chile. ${ }^{7}$ Department of Physiology, School of Medicine, Universidad Austral de Chile, Valdivia, Chile.

${ }^{8}$ Center for Interdisciplinary Studies of the Nervous System (CISNe), Universidad Austral de Chile, Valdivia, Chile.

Keywords: TDP-43, TARDBP, retrotransposition, RNA biogenesis, RNAbinding protein

*Correspondence:

Juan C. Opazo

jopazo@gmail.com

Gonzalo A. Mardones

gonzalo.mardones@uach.cl 


\section{Abstract}

36 The TAR DNA Binding Protein (TARDBP) gene has gained attention in

37 biomedicine after the discovery of several pathogenic mutations. The lack of

38 knowledge about its evolutionary history contrasts with a large number of

39 studies in the biomedical area. This study aimed to investigate the

40 retrotransposition evolutionary dynamics associated with this gene in

41 primates. We identified retropseudogenes that originated in the ancestors of

42 anthropoids, catarrhines, and lemuriformes, i.e. the strepsirrhine clade that

43 inhabit Madagascar. We also found species-specific retropseudogenes in the

44 philippine tarsier, Bolivian squirrel monkey, capuchin monkey and vervet.

45 Although retropseudogenes are not able to produce a functional protein, we

46 can not rule out that they may represent genetic material upon which

47 evolution acts on, especially with regulatory functions. 


\section{Introduction}

49 Today, the availability of whole-genome sequences allows performing more detailed research on the evolution of different genetic elements. An important source of evolutionary innovation are the events of RNA retrotranscription and its insertion into the genome (Kaessmann et al. 2009; Casola \& Betrán 2017). These events depend on the enzymatic machinery encoded by retrotransposable elements, and they produce an intronless gene duplicate that could produce a protein similar to the parental gene (Zhang 2003). However, most retrotranscribed sequences are inserted at a random position in the genome, lacking all necessary transcription elements and becoming a pseudogene, a phenomenon called "dead on arrival" (Zhang 2003). However, retroseudogenes are not necessarily a dead end. There are cases in which they encode small interfering RNAs with regulatory functions (Tam et al. 2008; Watanabe et al. 2008). Thus, identifying the presence of retrocopies/retroseudogenes is an important piece of information to have a complete picture of the evolution of any particular gene.

The TAR DNA Binding Protein (TARDBP) gene, which encodes the Transactive response DNA-binding protein $43 \mathrm{kDa}$ (TDP-43), has gained considerable attention after the initial discovery that its mutations can cause familial amyotrophic lateral sclerosis (ALS) and frontotemporal dementia (FTD), two major forms of neurodegenerative disorders (Sreedharan et al. 2008; Kabashi et al. 2008). Up to date, more than 50 pathogenic missense mutations have been characterized (Sreedharan et al. 2008; Kabashi et al. 2008). TDP-43 is an RNA-binding protein with a variety of RNA metabolism functions, including transcription, mRNA transport and stabilization, miRNA biogenesis, long noncoding RNA processing, and translation (Hanson et al. 2012). More recent findings indicate that TDP-43 participates in the pathogenesis of other neurodegenerative disorders of several other proteinopathies, such as Parkinson's disease and Alzheimer's disease, which are conditions characterized by toxic protein aggregation (Klim et al. 2021). In human cells, under physiological conditions, TDP-43 mainly localizes in the nucleus, but in neurons and glial cells of ALS and FTD patients it shuttles and accumulates in the cytoplasm where eventually aggregates and contribute to the onset and progression of these diseases (Neumann et al. 2006; Arai et al. 2006; Robberecht \& Philips 2013; Heyburn \& Moussa 2017;

83 Pinarbasi et al. 2018). The TARDBP gene is conserved in species that share a

84 common ancestor deep in time (Wang et al. 2004), suggesting that this

85 gene carries out essential functions. This gene underwent an event of 
positive selection in the ancestor of mammals (Zhao et al. 2020), suggesting functional adaptations for the group. More recently in evolutionary time, it has been shown that during the evolution of humans, genes related to diseases like Alzheimer's also underwent positive selection (Vamathevan et al. 2008). Although events of positive selection are seen as conferring selective advantage, as a by-product, they can also have adverse effects (Holt et al. 1996). In this regard, it is proposed that human susceptibility to neurodegenerative disorders could be a consequence of improving our cognitive function (Gearing et al. 1994; Keller \& Miller 2006).

The aim of this study is to investigate the retrotransposition dynamics associated with the TARDBP gene in primates. According to our phylogenetic and synteny analyses, we identified retropseudogenes that originated at different times during the evolution of primates. TARDBP retropseudogenes originated in the anthropoid ancestor, between 67 and 43.2 million years ago, in the ancestor of catarrhines, between 43.2 and 29.44 million years ago, and in the ancestor of lemuriformes, i.e. the strepsirrhine clade that inhabit madagascar, between 59.3 and 55 million years ago. We also found species-specific retropseudogenes in the philippine tarsier (Carlito syrichta), Bolivian squirrel monkey (Saimiri boliviensis), capuchin monkey (Cebus capucinus imitator) and vervet (Chlorocebus sabaeus). Although annotated sequences are not putatively functional, given the presence of indels and premature stop codons, we can not rule out that these retrotransposed sequences may represent material on which evolution acts, especially to regulate the expression of their parental gene.

\section{Results}

\section{Multiple retropseudogenes lineages characterize the evolution of the} TARDBP gene in primates.

According to our phylogenetic and synteny analyses, we identified retropseudogenes of the TARDBP gene that originated at different times during the evolution of primates. We identified retropseudogenes originated in the ancestor of anthropoids, between 67 and 43.2 million years ago, in the ancestor of catarrhines, between 43.2 and 29.44 million years ago, and in the ancestor of lemuriformes, i.e. the strepsirrhine clade that inhabit madagascar, between 59.3 and 55 million years ago (Fig. 1). More recently in evolutionary time, we found species-specific retropseudogenes in the philippine tarsier (Carlito syrichta), Bolivian squirrel monkey (Saimiri boliviensis), capuchin monkey (Cebus capucinus imitator) and vervet 
(Chlorocebus sabaeus). All of them did not have intron sequences and were identified on a different autosome in comparison to the chromosomal location of the functional copy (Fig. 1). Our gene tree did not significantly deviate from the most updated phylogenetic hypotheses for the main group of primates (Pozzi et al. 2014; Finstermeier et al. 2013; Perelman et al. 2011), suggesting that the functional copy of the TARDBP gene was present as a simple copy gene in the ancestor of the group (Fig. 1).

We recovered three highly supported monophyletic groups containing representative species of all major groups of anthropoids i.e. apes, Old World monkeys and New World monkeys (light blue, brown and green lineages, Fig. 1), indicating that these retropseudogenes originated in the ancestor of the group, between 67 and 43.2 millions of years ago, and were maintained in representative species of all descendant primate groups. The retropseudogene lineage depicted with the purple shading (Fig. 1), although it was not recovered monophyletic, our synteny analyses suggest that it indeed belongs to a single lineage (Fig. 1). Representative species of the three purple clades possess the same flanking genes, DIAPH3 at the 5 ' side and TDRD3 at the $3^{\prime}$ side of the retropseudogene, strongly suggesting that the lack of monophyly could be attributed to a phylogenetic artifact (Fig. 1). The small number of changes, as illustrated by the short branches that define the sister group relationships of the main clades, could be the main cause (Fig. 1). We also found a retropseudogene lineage that according to our phylogenetic tree originated in the ancestor of catarrhine primates, the group that includes apes and Old World monkeys (blue lineage, Fig. 1), between 43.2 and 29.44 million years ago. In this case, we recovered a clade containing the functional copy of the TARDBP gene in catarrhines (upper pink lineage, Fig. 1), sister to a group containing a retropseudogene in the same primate group (blue lineage, Fig. 1). The clade containing TARDBP functional sequences from New World monkeys was recovered sister to the above mentioned clade (Fig. 1). In this clade in addition to the functional TARDBP copy, we found New World monkey specific retropseudogenes for which the evolutionary history is difficult to resolve given the shortness of the branches (Fig. 1). We identified three retropseudogenes, two in the capuchin monkey (Cebus capucinus imitator) and one in the Bolivian squirrel monkey (Saimiri boliviensis). Finally, we recovered a sequence from the Angola colobus (Colobus angolensis)(yellow branch, Fig. 1), which was recovered sister to a clade containing the TARDBP functional copy (pink lineage, Fig. 1) and three retropseudogenes lineages 
162 (blue, purple and green clades, Fig. 1). The phylogenetic position of this 163 branch in our gene tree suggests that it represents a retropseudogene 164 originated in the anthropoid ancestor, but only conserved in this species. In 165 support of this claim, the single flanking gene (PRMT2) found in the genomic 166 piece containing the TARDBP retropseudogene in the Angola colobus is not shared with any other gene lineage described in this study (Fig. 1). In all cases, the identified retropseudogenes during the evolutionary history of anthropoid primates have premature stop codons and indels (supplementary figures 1 to 5 ).

We also identified retropseudogenes in tarsiers and strepsirrhines (Fig.

172 1). We found a single retropseudogene in the Philippine tarsier (Carlito

173 syrichta), which shows the hallmark of a sequence free from selective 174 constraints, i.e., a long branch as a signal of an accelerated rate of evolution 175 in comparison to the functional copy (Fig. 1). In the strepsirrhine clade we identified a highly supported lineage containing the TARDBP functional copy in three species, greater bamboo lemur (Prolemur simus), coquerel's sifaka (Propithecus coquereli) and the mouse lemur (Microcebus murinus), which in turn was recovered sister to an also highly supported clade containing retropseudogenes in the greater bamboo lemur (Prolemur simus) and coquerel's sifaka (Propithecus coquereli) (Fig.1). This tree topology suggests that this retrocopy originated in the ancestor of lemuriformes, i.e. the strepsirrhine clade that inhabits Madagascar, between 59.3 and 55 million years ago, and it has been maintained in the genome of descendant species. Finally, the functional copy of the bushbaby (Otolemur garnettii) was recovered sister to the lemuriformes clade. Similar to the case of anthropoids, all retropseudogenes identified in tarsiers and strepsirrhines have premature stop codons and indels (supplementary figures 6 and 7).

\section{Discussion}

In this study we revealed that the evolutionary history of TARDBP, an RNAbinding protein involved in several human neurodegenerative disorders (Sreedharan et al. 2008; Kabashi et al. 2008), is characterized by the presence of retropseudogenes that originated at different ages during the evolutionary history of primates. An important fraction of the retropseudogenes originated in the anthropoid ancestor, between 67 and 43.2 million years ago, and has remained in the genome of the species (Fig.

198 2). This phenomenon fits the expectation of a peak of retrocopy formation 
200 retroelements that produced an explosion in SINE/Alu repeat amplification (Ohshima et al. 2003; Marques et al. 2005). Interestingly, this period of time represents a key moment during the evolutionary history of primates, the radiation of the anthropoid lineage, where significant morphological and physiological traits arouse (Kay et al. 1997).

Although we did not find retrocopies, i.e. intronless copies with the potential to produce a similar protein compared to the parental gene, it has been suggested that retropseudogenes could encode small interfering RNAs that can regulate the expression of their parental genes (Tam et al. 2008; Watanabe et al. 2008). Thus, this period of Vesuvian mode of evolution could be seen as a source of evolutionary novelty that fueled the origin of the phenotypes that define the anthropoid lineage (Long et al. 2003; Kaessmann et al. 2009; Casola \& Betrán 2017). Other retropseudogenes originated in the catarrhine ancestor, between 43.2 and 29.44 million years ago, and in other primate groups (Fig. 2).

In agreement with the literature, and given the nature of the process originating retrocopies, all of them seem to be non-functional as canonical TARDBP (Zhang 2003), which can be verified by the presence of indels and premature stop codons (supplementary figures 1 and 7). The identification of several retropseudogenes for the TARDBP gene in primates appears to be not a surprise as this gene comply all the requisites to be a gene with multiple retropseudogenes (Zhang \& Gerstein 2004; McDonell \& Drouin 2012; Gonçalves et al. 2000), i.e., short transcripts (61 to 414 amino acids)(Yates et al. 2020), widely and highly expressed (Uhlén et al. 2015), low GC-content (47\%, average among 23 primate species) and highly conserved (3.37\%, maximal divergence among primates). Furthermore, in agreement with slow rate of length abridgment, the identified retropseudogenes possess a length (mean 1128 bp, median 1193 bp) similar to the functional TARDBP gene (1245 bp).

Among apes, the number of TARDBP retrotransposition events appear to be higher in comparison to the average number of retrocopies per

231 parental gene in their genomes (Navarro \& Galante 2015). On average, ape 232 genomes possess 2.91 retrocopies per parental gene (Navarro \& Galante 233 2015), however in our study we identified five TARDBP retropseudogenes in 234 each examined ape species. Coincident with previous evidence, we also 235 found a higher number of retropseudogenes in New World monkeys (Navarro 236 \& Galante 2015). Although it is not clear the reason why this primate group 237 possesses more retrocopies in comparison to catarrhines, it is suggested 
238 that a lineage specific expansion of L1PA1 and L1P3 subelements could be linked to the observed pattern (Navarro \& Galante 2015; Casola \& Betrán 2017).

In conclusion, in this work, we demonstrate that the TARDBP gene in primates has an evolutionary history characterized by the presence of multiple retropseudogene lineages. In the ancestor of anthropoids occurred a burst of activity, which led to intronless sequences that can not give rise to functional proteins. However, we can not discard that these DNA retrotransposed sequences could represent the raw genetic material for the evolution to act on, especially to regulate the expression of their parental gene, as it has been described for other genes.

\section{Material and Methods}

\section{DNA sequences}

253 We performed searches for TAR DNA Binding Protein (TARDBP) genes in primate genomes in Ensembl v.102 (Yates et al. 2020). We retrieved primate orthologs, using the human (Homo sapiens) entry, based on the ortholog prediction function of Ensembl v.102 (Yates et al. 2020). We identified TARDBP retropseudogenes in primate species by performing BLASTN searches (Altschul et al. 1990), against the whole genome sequence in Ensembl v.102 (Yates et al. 2020) using default settings. In each case the query sequence (TARDBP) was from the same species of the genome in which retropseudogenes were looking for. In our searches, a retropseudogene was recognized as a sequence containing all exons together and found in a different chromosome in comparison to the functional copy. Genomic fragments containing retropseudogenes were extracted and manually annotated by comparing the coding sequence of the same species using the program Blast2seq v2.5 (Tatusova \& Madden 1999) with default parameters. Accession numbers and details about the taxonomic sampling are available in Supplementary Table S1.

Nucleotide sequences were aligned using MAFFT v.7 (Katoh \& Standley 2013), allowing the program to choose the alignment strategy (FFT-NS-i). We used the proposed model tool of IQ-Tree v.1.6.12 (Kalyaanamoorthy et al. 2017) to select the best-fitting model of nucleotide substitution, which selected GTR+F+R3. We used the maximum likelihood method to obtain the best tree using the program IQ-Tree v1.6.12 (Trifinopoulos et al. 2016). We assessed support for the nodes using three strategies: a Bayesian-like 
276 transformation of aLRT (aBayes test) (Anisimova et al. 2011), SH-like 277 approximate likelihood ratio test (SH-aLRT) (Guindon et al. 2010) and the 278 ultrafast bootstrap approximation (Hoang et al. 2018). We carried out 20 279 independent runs to explore the tree space, and the tree with the highest 280 likelihood score was chosen. TARDBP sequences from the African elephant 281 (Loxodonta africana), blue whale (Balaenoptera musculus) and red fox 282 (Vulpes vulpes) were used as outgroups.

285 We examined genes found upstream and downstream of functional copies and retropseudogenes. We used the estimates of orthology and paralogy derived from the Ensembl Compara database (Herrero et al. 2016); these estimates are obtained from a pipeline that considers both synteny and phylogeny to generate orthology mappings. These predictions were visualized using the program Genomicus v100.01 (Nguyen et al. 2018). Our assessments were performed in representative species for each lineage.

\section{Acknowledgements}

294 This work was supported by Fondo Nacional de Desarrollo Científico y

295 Tecnológico from Chile (FONDECYT 1210471) and Millennium Nucleus of Ion

296 Channels Associated Diseases (MiNICAD), Iniciativa Científica Milenio, 297 Ministry of Economy, Development and Tourism from Chile to JCO, Fondo 298 Nacional de Desarrollo Científico y Tecnológico from Chile (FONDECYT $2991180957)$ to FJM and LVC and Fondo Nacional de Desarrollo Científico y 300 Tecnológico from Chile (FONDECYT 1211481) to GAM.

\section{Author contributions}

303 JCO and GAM designed the study. KZ, JCO collected and analyzed data. JCO 304 and GAM wrote the manuscript. LV-C, FJM reviewed and edited the 305 manuscript. All authors contributed to the article and approved the submitted 306 version.

\section{Competing Interests}

309 The authors declare no competing of interest 


\section{Figure legends}

311 Figure 1. Maximum likelihood tree showing sister group relationships 312 between the functional copy of TARDBP and primate retropseudogenes. 313 Numbers on the nodes correspond to support values from the ultrafast 314 bootstrap routine. TARDBP sequences from the African elephant (Loxodonta 315 africana), blue whale (Balaenoptera musculus) and red fox (Vulpes vulpes) 316 were used as outgroups (not shown). The scale denotes substitutions per site 317 and colors represent lineages. The pink lineage represents the TARDBP 318 functional copy. Synteny information is provided for each lineage at the right 319 side of the figure.

321 Figure 2. Time calibrated primate phylogeny showing the origin of the 322 different retropseudogene lineages depicted in figure 1 . Numbers on the 323 triangles correspond to retropseudogenes originated within a particular group 324 of primates. Silhouette images were obtained from PhyloPic 325 (http://phylopic.org/). Divergence times were obtained from timetree 326 (http://www.timetree.org/)(Kumar et al. 2017). 


\section{References}

Altschul SF, Gish W, Miller W, Myers EW, Lipman DJ. 1990. Basic local alignment search tool. J. Mol. Biol. 215:403-410.

Anisimova M, Gil M, Dufayard J-F, Dessimoz C, Gascuel O. 2011. Survey of branch 332 support methods demonstrates accuracy, power, and robustness of fast likelihood333 based approximation schemes. Syst. Biol. 60:685-699.

334 Arai T et al. 2006. TDP-43 is a component of ubiquitin-positive tau-negative 335 inclusions in frontotemporal lobar degeneration and amyotrophic lateral sclerosis.

336 Biochem. Biophys. Res. Commun. 351:602-611.

337 Casola C, Betrán E. 2017. The Genomic Impact of Gene Retrocopies: What Have We Learned from Comparative Genomics, Population Genomics, and Transcriptomic Analyses? Genome Biol. Evol. 9:1351-1373.

340 Finstermeier $\mathrm{K}$ et al. 2013. A mitogenomic phylogeny of living primates. PLoS One. 341 8:e69504.

Gearing M, Rebeck GW, Hyman BT, Tigges J, Mirra SS. 1994. Neuropathology and apolipoprotein $\mathrm{E}$ profile of aged chimpanzees: implications for Alzheimer disease. Proc. Natl. Acad. Sci. U. S. A. 91:9382-9386.

Gonçalves I, Duret L, Mouchiroud D. 2000. Nature and structure of human genes that generate retropseudogenes. Genome Res. 10:672-678.

347 Guindon S et al. 2010. New algorithms and methods to estimate maximum348 likelihood phylogenies: assessing the performance of PhyML 3.0. Syst. Biol. 59:307-321.

Hanson KA, Kim SH, Tibbetts RS. 2012. RNA-binding proteins in neurodegenerative disease: TDP-43 and beyond. Wiley Interdiscip. Rev. RNA. 3:265-285.

Herrero J et al. 2016. Ensembl comparative genomics resources. Database . 2016.

354 Heyburn L, Moussa CE-H. 2017. TDP-43 in the spectrum of MND-FTLD pathologies. 355 Mol. Cell. Neurosci. 83:46-54.

356 Hoang DT, Chernomor O, von Haeseler A, Minh BQ, Vinh LS. 2018. UFBoot2:

357 Improving the Ultrafast Bootstrap Approximation. Mol. Biol. Evol. 35:518-522.

358 Holt RD, Nesse RM, Williams GC. 1996. Why We Get Sick: The New Science of 359 Darwinian Medicine. Ecology. 77:983. doi: 10.2307/2265522.

360 Kabashi $\mathrm{E}$ et al. 2008. TARDBP mutations in individuals with sporadic and familial 361 amyotrophic lateral sclerosis. Nat. Genet. 40:572-574.

362 Kaessmann H, Vinckenbosch N, Long M. 2009. RNA-based gene duplication: 363 mechanistic and evolutionary insights. Nat. Rev. Genet. 10:19-31. 
Kalyaanamoorthy S, Minh BQ, Wong TKF, von Haeseler A, Jermiin LS. 2017. ModelFinder: fast model selection for accurate phylogenetic estimates. Nat. Methods. 14:587-589.

367 Katoh K, Standley DM. 2013. MAFFT multiple sequence alignment software version 368 7: improvements in performance and usability. Mol. Biol. Evol. 30:772-780.

369 Kay RF, Ross C, Williams BA. 1997. Anthropoid origins. Science. 275:797-804.

370 Keller MC, Miller G. 2006. Resolving the paradox of common, harmful, heritable mental disorders: which evolutionary genetic models work best? Behav. Brain Sci. 29:385-404; discussion 405-52.

373 Klim JR, Pintacuda G, Nash LA, Guerra San Juan I, Eggan K. 2021. Connecting TDP37443 Pathology with Neuropathy. Trends Neurosci. doi: 10.1016/j.tins.2021.02.008.

375 Kumar S, Stecher G, Suleski M, Hedges SB. 2017. TimeTree: A Resource for 376 Timelines, Timetrees, and Divergence Times. Mol. Biol. Evol. 34:1812-1819.

377 Long M, Betrán E, Thornton K, Wang W. 2003. The origin of new genes: glimpses 378 from the young and old. Nat. Rev. Genet. 4:865-875.

379 Marques AC, Dupanloup I, Vinckenbosch N, Reymond A, Kaessmann H. 2005. 380 Emergence of young human genes after a burst of retroposition in primates. PLoS 381 Biol. 3:e357.

McDonell L, Drouin G. 2012. The abundance of processed pseudogenes derived from glycolytic genes is correlated with their expression level. Genome. 55:147384151.

385 Navarro FCP, Galante PAF. 2015. A Genome-Wide Landscape of Retrocopies in Primate Genomes. Genome Biol. Evol. 7:2265-2275.

Neumann $\mathrm{M}$ et al. 2006. Ubiquitinated TDP-43 in frontotemporal lobar degeneration and amyotrophic lateral sclerosis. Science. 314:130-133.

389 Nguyen NTT, Vincens P, Roest Crollius H, Louis A. 2018. Genomicus 2018:

390 karyotype evolutionary trees and on-the-fly synteny computing. Nucleic Acids Res.

391 46:D816-D822.

392 Ohshima $\mathrm{K}$ et al. 2003. Whole-genome screening indicates a possible burst of 393 formation of processed pseudogenes and Alu repeats by particular L1 subfamilies in 394 ancestral primates. Genome Biol. 4:R74.

395 Perelman P et al. 2011. A molecular phylogeny of living primates. PLoS Genet. 396 7:e1001342.

397 Pinarbasi ES et al. 2018. Active nuclear import and passive nuclear export are the 398 primary determinants of TDP-43 localization. Sci. Rep. 8:7083.

399 Pozzi L et al. 2014. Primate phylogenetic relationships and divergence dates 
400 inferred from complete mitochondrial genomes. Mol. Phylogenet. Evol. 75:165-183.

401 Robberecht W, Philips T. 2013. The changing scene of amyotrophic lateral sclerosis. 402 Nat. Rev. Neurosci. 14:248-264.

403 Sreedharan J et al. 2008. TDP-43 mutations in familial and sporadic amyotrophic 404 lateral sclerosis. Science. 319:1668-1672.

405 Tam $\mathrm{OH}$ et al. 2008. Pseudogene-derived small interfering RNAs regulate gene 406 expression in mouse oocytes. Nature. 453:534-538.

407 Tatusova TA, Madden TL. 1999. BLAST 2 Sequences, a new tool for comparing 408 protein and nucleotide sequences. FEMS Microbiol. Lett. 174:247-250.

409 Trifinopoulos J, Nguyen L-T, von Haeseler A, Minh BQ. 2016. W-IQ-TREE: a fast 410 online phylogenetic tool for maximum likelihood analysis. Nucleic Acids Res. 411 44:W232-5.

412 Uhlén M et al. 2015. Proteomics. Tissue-based map of the human proteome. 413 Science. 347:1260419.

414 Vamathevan J] et al. 2008. The role of positive selection in determining the 415 molecular cause of species differences in disease. BMC Evol. Biol. 8:273.

416 Wang H-Y, Wang I-F, Bose J, Shen C-KJ. 2004. Structural diversity and functional 417 implications of the eukaryotic TDP gene family. Genomics. 83:130-139.

418 Watanabe T et al. 2008. Endogenous siRNAs from naturally formed dsRNAs regulate 419 transcripts in mouse oocytes. Nature. 453:539-543.

420 Yates AD et al. 2020. Ensembl 2020. Nucleic Acids Res. 48:D682-D688.

421 Zhang J. 2003. Evolution by gene duplication: an update. Trends Ecol. Evol. 422 18:292-298.

423 Zhang Z, Gerstein M. 2004. Large-scale analysis of pseudogenes in the human 424 genome. Curr. Opin. Genet. Dev. 14:328-335.

425 Zhao L et al. 2020. TDP-43 facilitates milk lipid secretion by post-transcriptional 426 regulation of Btn1a1 and Xdh. Nat. Commun. 11:341. 
bioRxiv preprint doi: https://doi.org/10.1101/2021.05.24.445516; this version posted May 25, 2021. The copyright holder for this preprint (which was not certified by peer review) is the author/funder, who has granted bioRxiv a license to display the preprint in perpetuity. It is made available under aCC-BY 4.0 International license.

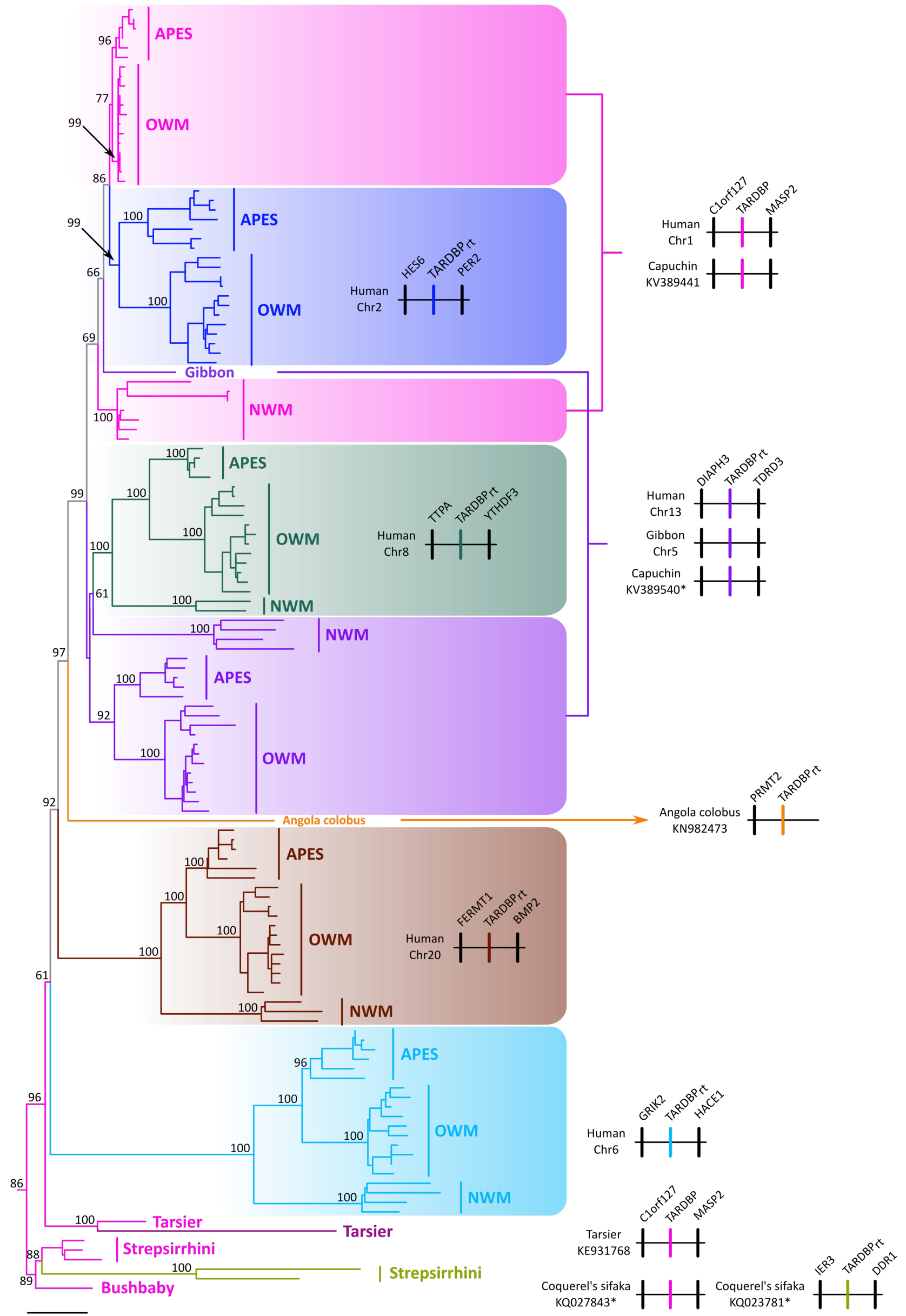



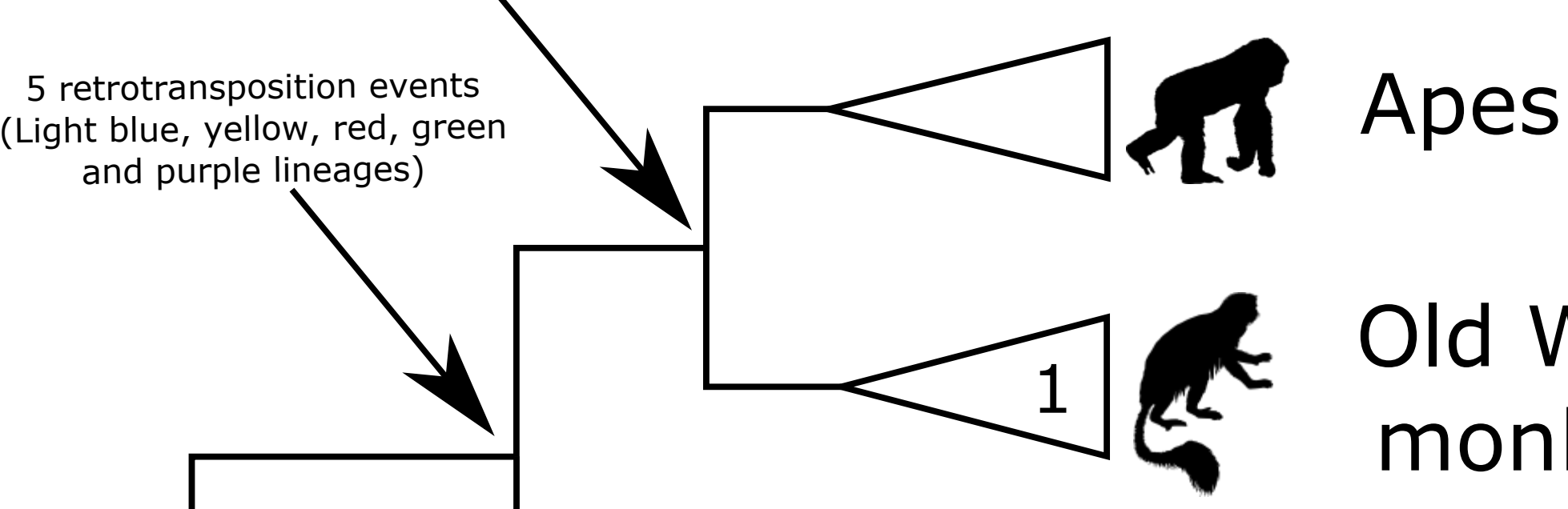

Old World monkeys

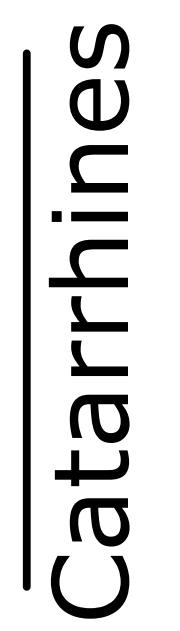
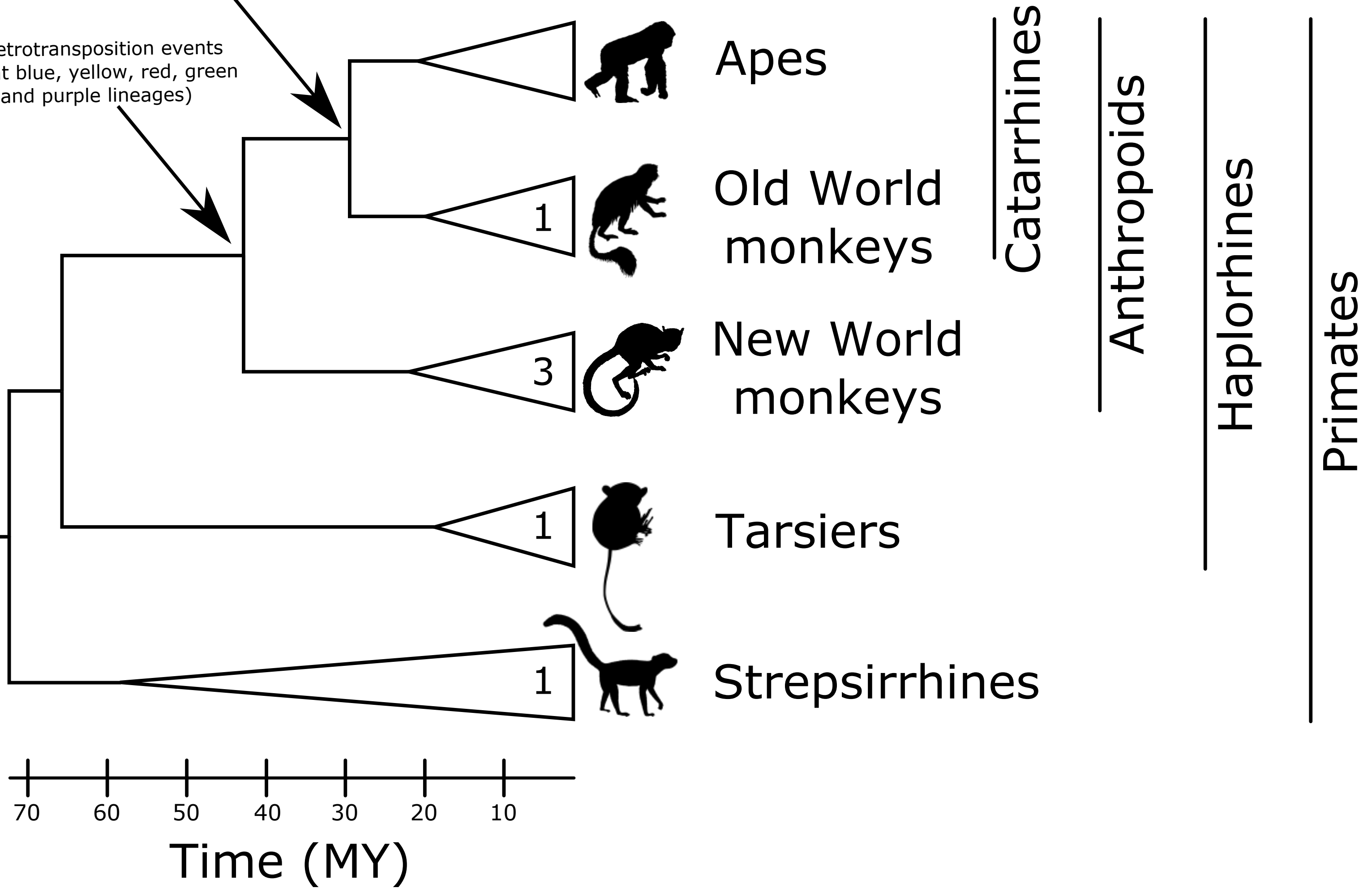

Tarsiers 
Human Chr1 TARDBP

Human Chr2

Macaque Chr1

Human Chr1 TARDB

Human Chr2

Macaque Chr12

Human Chr1 TARDBP

Human Chr2

Macaque Chr12

Human Chr1 TARDBP

Human Chr2

Macaque Chr12
1 A TCT 10

$M \quad S \quad F \quad Y$ T A C G GTA ACC GA GAT GAG AAC GAT GAG CCC ATT GAA ATA CCA TCG GAA GAC GAT

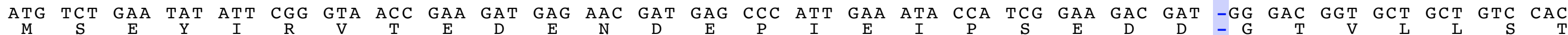

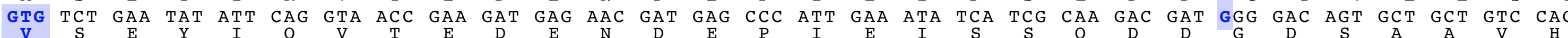

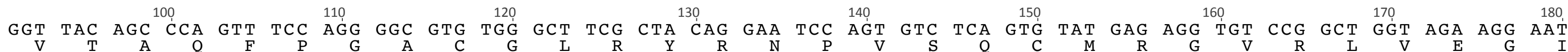

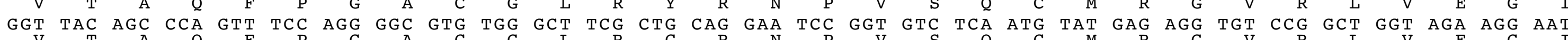
GCT TAT GGC ACA GTT TCC AGG GGC GTG TGG GCT TCG CTA CAG AAA TCC AGC GTC TCA ATG TAT GAG AGG TGT ACG GCT GGT AGA AGG AAT

190 220

230

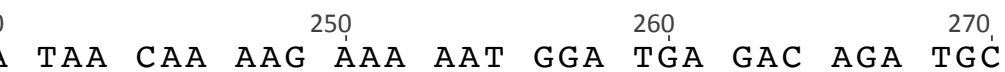

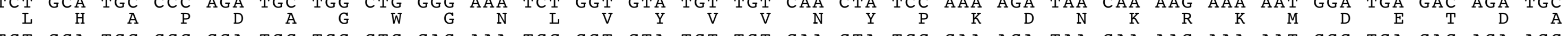

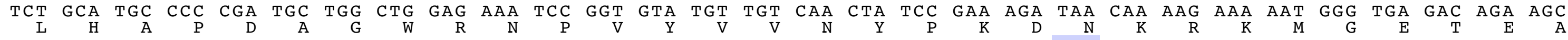



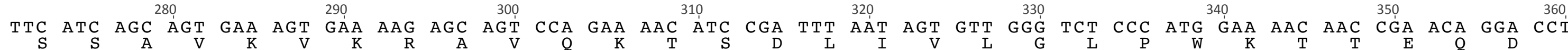

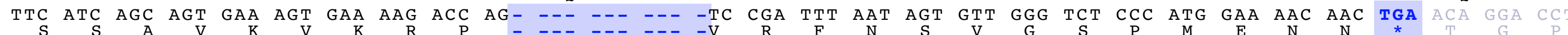



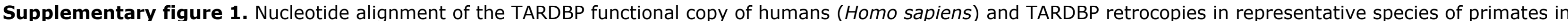

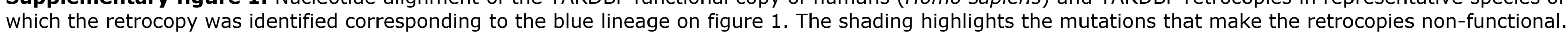


Macaque Chr17

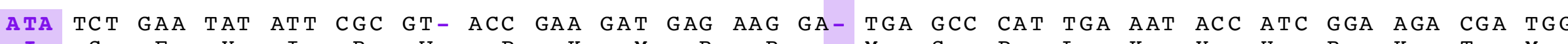

Marmoset NTIC01000001

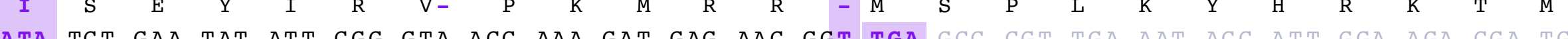

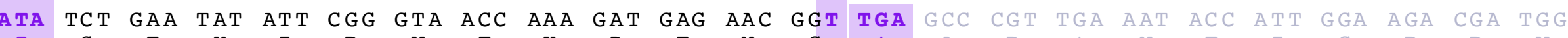

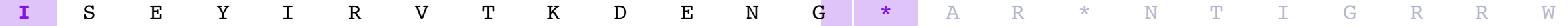

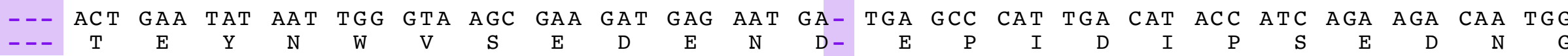

Human Chr1 TARDBP

Human Chr13

Macaque Chr17

Marmoset NTIC01000001

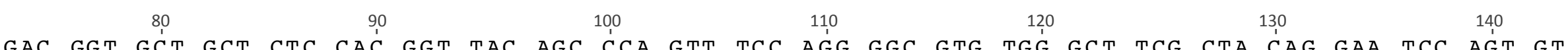

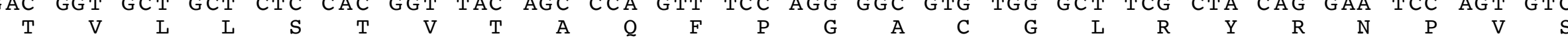

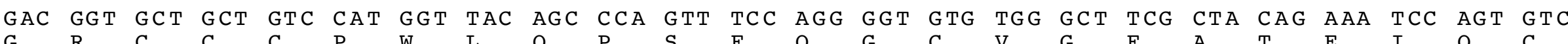

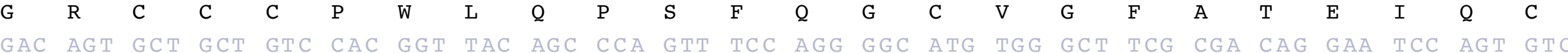



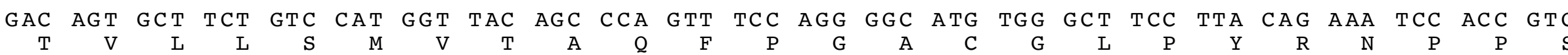

Human Chr1 TARDBP

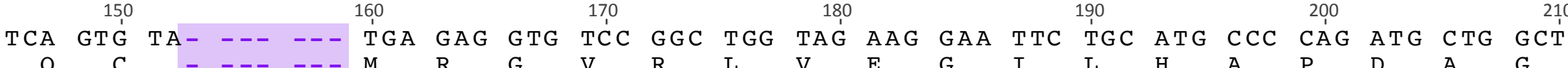
190 2000 TCA GTG TA- --- -- TGA GAG GTG TCC GGT TGG TAG AAG GAA TTC TTC ATG CCC CCG ATG CTG GCT L S V V - - - - * * E V V S G G

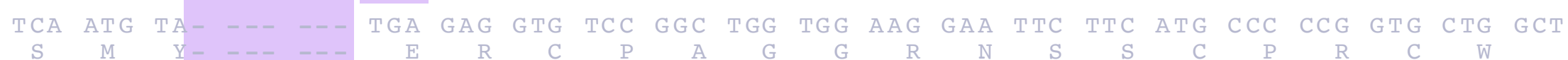
Macaque Chr17

Marmoset NTIC01000001


$\begin{array}{llllllllllllllllllllll}\mathrm{Q} & \mathrm{C} & \mathrm{M} & \mathrm{R} & \mathrm{G} & \mathrm{E} & \mathrm{R} & \mathrm{C} & \mathrm{P} & \mathrm{T} & \mathrm{G} & \mathrm{R} & \mathrm{R} & \mathrm{N} & \mathrm{A} & \mathrm{A} & \mathrm{C} & \mathrm{P} & * & \mathrm{C} & \mathrm{W}\end{array}$

Supplementary figure 2. Nucleotide alignment of the TARDBP functional copy of humans (Homo sapiens) and TARDBP retrocopies in representative species of primates in which the retrocopy was identified corresponding to the purple lineage on figure 1 . The shading highlights the mutations that make the retrocopies non-functional. 


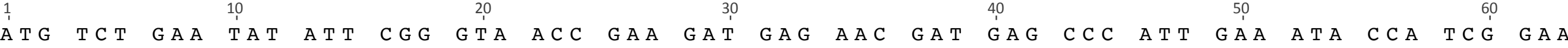

Human Chr8

Macaque Chr8

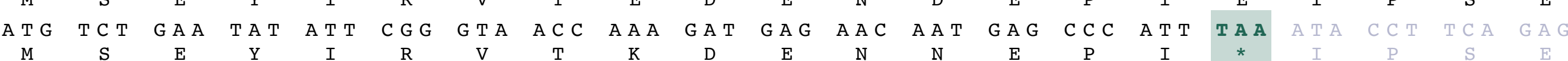

Ma's night monkey KZ195588

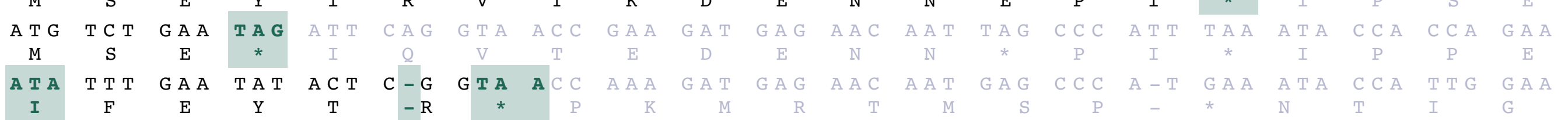

Human Chr1 TARDBP

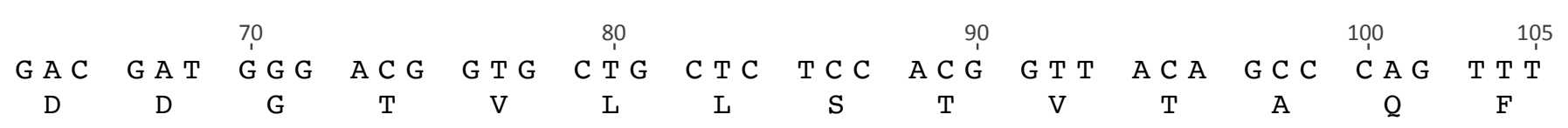

Human Chr8

Macaque Chr8

Ma's night monkey KZ195588

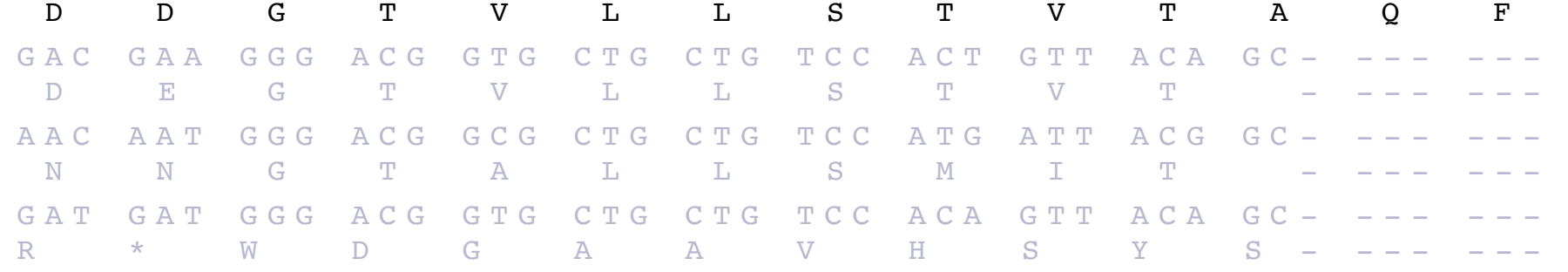

Supplementary figure 3. Nucleotide alignment of the TARDBP functional copy of humans (Homo sapiens) and TARDBP retrocopies in representative species of primates in which the retrocopy was identified corresponding to the green lineage on figure 1 . The shading highlights the mutations that make the retrocopies non-functional. 
Human Chr1 TARDBP

Human Chr20

Macaque Chr10

Marmoset NTIC01034389

Human Chr1 TARDBP

Human Chr20

Macaque Chr10

Marmoset NTIC01034389

Human Chr1 TARDBP

Human Chr20

Macaque Chr10

Marmoset NTIC01034389

Human Chr1 TARDBP

Human Chr20

Macaque Chr10

Marmoset NTIC01034389
$1020 \quad 10 \quad 30$ GAG AAC GAT 60

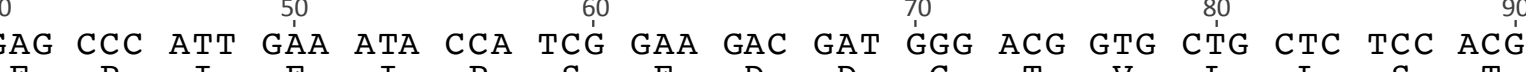
$\begin{array}{lllllllllllllllll} & P & I & E & I & P & S & E & D & D & G & T & V & L & L & S & T\end{array}$

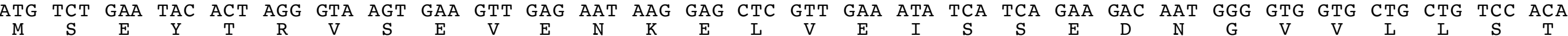
ATG TTT GAA TAC ACT TGG GTA AGT GAA GAT GAG AAT AAg GAg CTC ATG GAA ATA TCA TCA GAA TAC AAT GGG GTA GTG CTG CCG TCC ACA ATG TCT GA TAT ACT CAA GTA ACT GAA GAT GAG AAT AAG GAA CTC TTT GAA AT- -

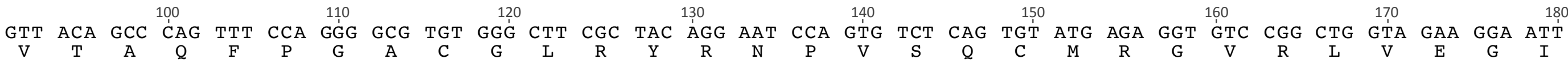

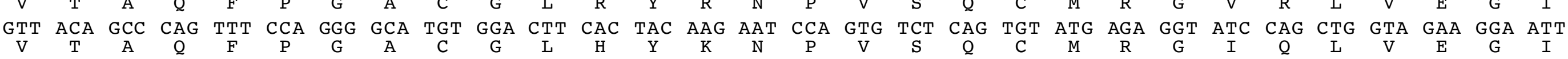
V



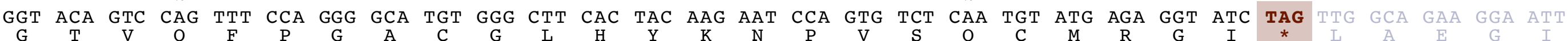

$\begin{array}{ccccccc}190 & 200 & 210 & 220 & 230 & 240 & 250\end{array}$ CTG CAT GCC CCA GAT GCT GGC TGG GGA AAT CTG GTG TAT GTT GTC AAC TÁ CCA AAA GAT AAC AAA AGA AAA ATG GAT GAG ACA GAT GCT

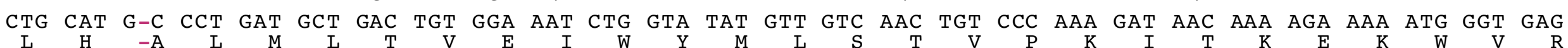

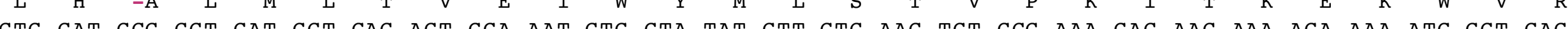

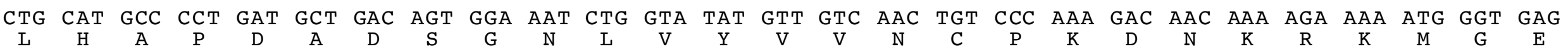
CTG CAT GCC CCT CAT GCT GCC TGT GGA AAT CTG GTC TAT GTT GTC AAC TAT C C A A A GCC AAC AAA AGA AAA ATG GAT GAG

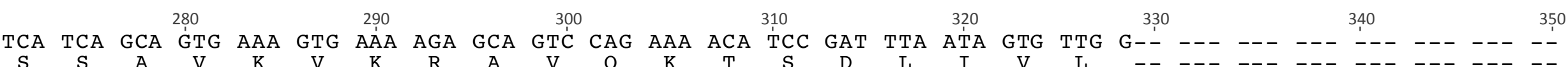
--- -CG GCA GTG AAA GTG --A AGA GCA GTC CAG AAA ATA TCT TAT TTA ATA GCG TTG GTA ATT AGT GAG TTT TTG CTC TC --- -

--- -CA GCA GTG AAA GTG --A AGA GCA GTC CAg AAA ATA TCT TAC TTA ATA GTA CTG GTA ATT AG $--_{-C A}$ TCA GTG AAA GTG - - A AGA GCA GCC CAG AAA ACA TCT TAT TTA ATA GTG TTG GTA ATT AGC

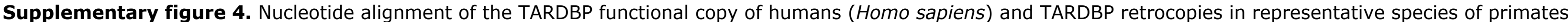

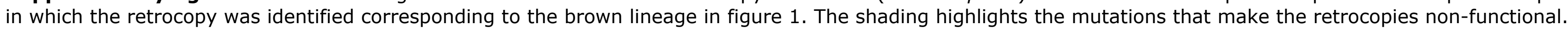




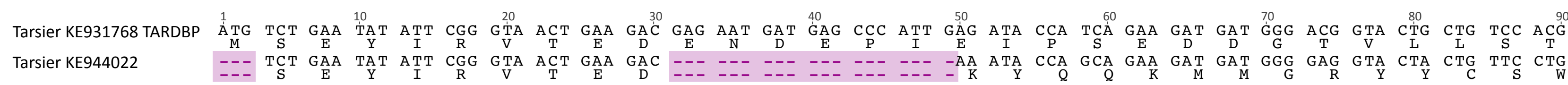

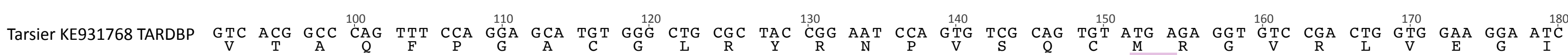

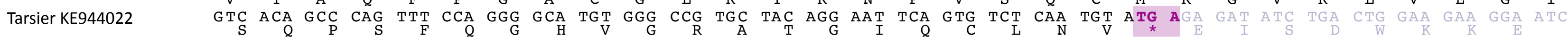

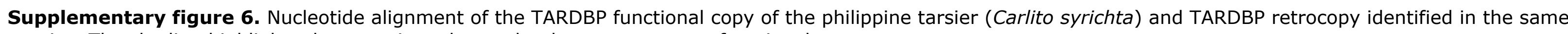
species. The shading highlights the mutations that make the retrocopy non-functional. 

Mouse lemur Chr2

Coquerel's sifaka KQ023781

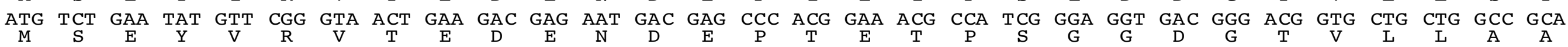

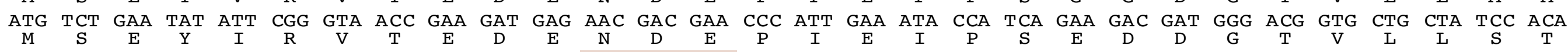

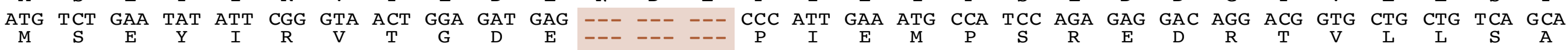





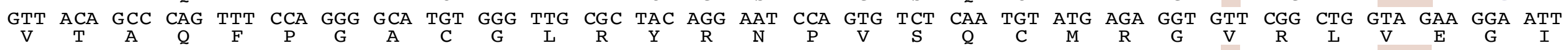

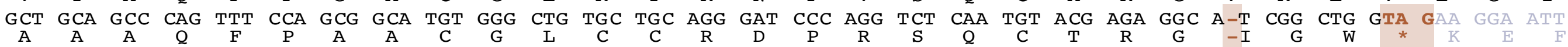

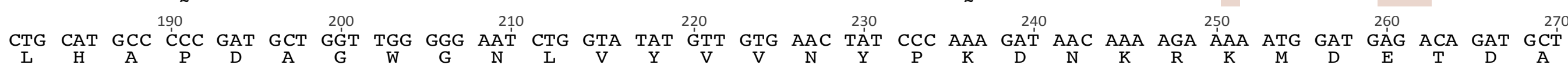

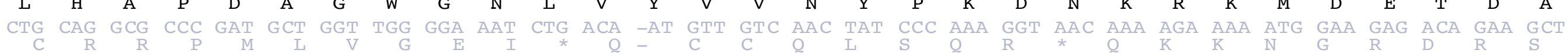

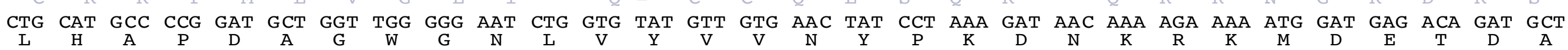

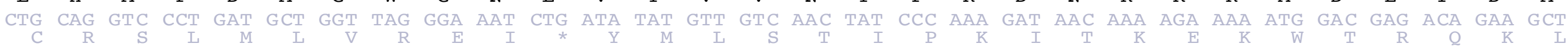



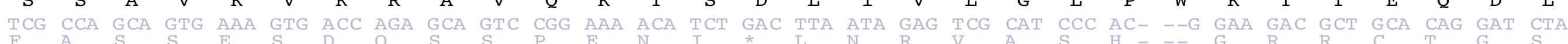

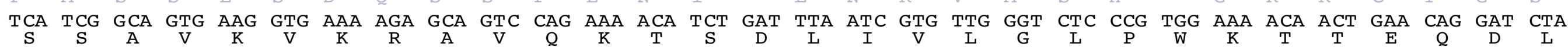
TCA CCA GCA GTG AAA GTG ACA AGA GCA GTC CGG AAA GCA TCT GAT TTA ATA GAG TTG CAT CCC C $\mathrm{C}-\ldots$


AAG GGA --- - A AGT ACC TTT GGA GAA GTT CTT ATG GTG CAG GTC AAG AAA GAT GTT AAA ATT CCT CAT TCA AAA GGG TTT GGC TTC ATT

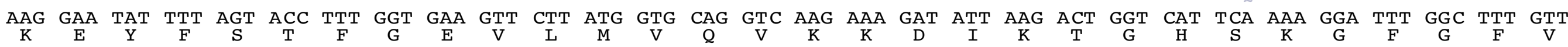

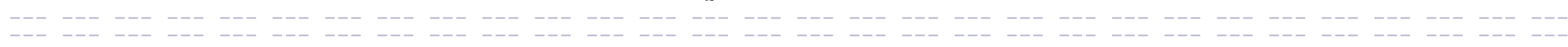

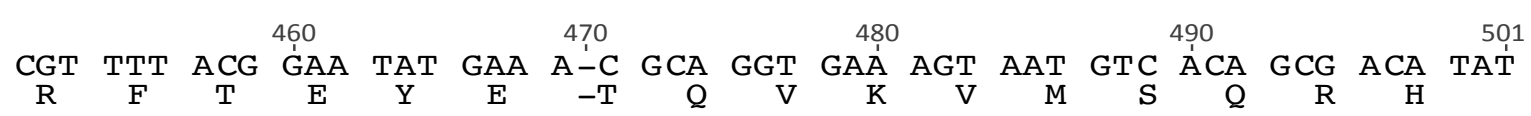

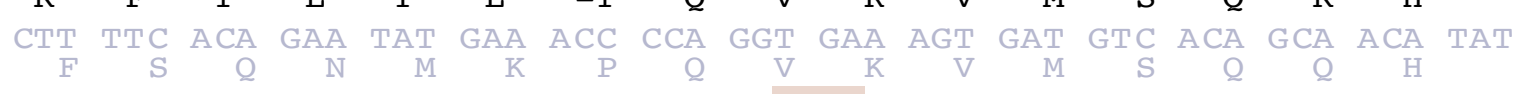



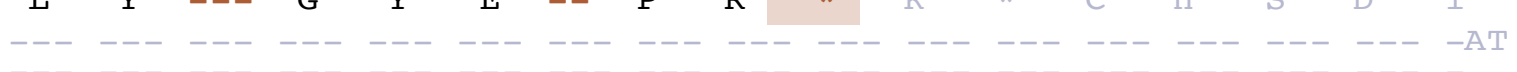
Coquerel's sifaka KQ023781

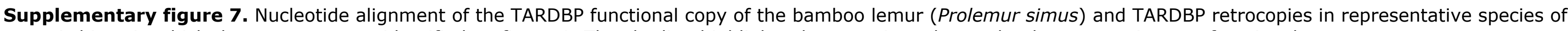
strepsirrhines in which the retrocopy was identified on figure 1 . The shading highlights the mutations that make the retrocopies non-functional. 